\title{
Impact of the Duration of Diabetes Mellitus on the Outcome of Metastatic Pancreatic Cancer Treated with Gemcitabine: A Retrospective Study
}

\author{
Sakura Iizumi ${ }^{1,2}$, Aya Kuchiba ${ }^{3}$, Takuji Okusaka ${ }^{1}$, Masafumi Ikeda ${ }^{4}$, Yasunari Sakamoto ${ }^{1}$, \\ Shunsuke Kondo ${ }^{1}$, Chigusa Morizane ${ }^{1}$, Hideki Ueno ${ }^{1}$, Keiichiro Osame ${ }^{5}$, Shuichi Mitsunaga ${ }^{4}$, \\ Izumi Ohno ${ }^{4}$, Hiroshi Imaoka ${ }^{4}$, Yusuke Hashimoto ${ }^{4}$, Hideaki Takahashi ${ }^{4}$, \\ Mitsuhito Sasaki ${ }^{4}$ and Ken Ohashi ${ }^{5}$
}

\begin{abstract}
:
Objective To assess the impact of the duration of diabetes mellitus (DM) on the outcomes of pancreatic cancer patients undergoing chemotherapy.

Methods We reviewed the medical records of patients with metastatic pancreatic cancer who received gemcitabine monotherapy as the standard therapy before the introduction of combination regimens. The treatment outcomes of gemcitabine were compared among three groups classified according to the duration of DM: no DM, short DM ( $<4$ years), and long DM ( $\geq 4$ years).

Results Among 350 patients, 218, 87, and 45 patients were classified into the no DM, short DM, and long DM groups, respectively. In comparison to the no DM group, the univariate hazard ratios (HRs) for progression-free survival (PFS) and overall survival (OS) were 1.44 [95\% confidence interval (CI), 1.02-2.02; $\mathrm{p}=0.027$ ] and 1.33 (95\% CI, 0.93-1.89; $\mathrm{p}=0.081)$, respectively, in the long DM group, and 1.12 (95\% CI, $0.85-1.46 ; \mathrm{p}=0.426)$ and 1.06 (95\% CI, 0.81-1.40; $\mathrm{p}=0.678)$, respectively, in the short DM group; the multivariate-adjusted HRs were $1.33(95 \% \mathrm{CI}, 0.94-1.89 ; \mathrm{p}=0.103)$ and 1.37 (95\% CI, 0.95-1.98; $\mathrm{p}=0.095)$ in the long DM group and 1.12 (95\% CI, 0.85-1.47; p=0.410) and 1.10 (95\% CI, 0.82-1.46; p=0.533) in the short DM group. The survival outcomes of the long DM group tended to remain poorer in analyses using different cutoffs of DM duration as, well as in hospital-specific analyses.

Conclusion Long-standing DM may be associated with shorter PFS and OS in patients with metastatic pancreatic cancer.
\end{abstract}

Key words: chemotherapy, diabetes mellitus, gemcitabine, pancreatic cancer

(Intern Med 58: 2435-2441, 2019)

(DOI: 10.2169/internalmedicine.2539-18)

\section{Introduction}

Diabetes mellitus (DM) is one of the most common comorbidities in cancer patients, particularly those with pancreatic cancer. While DM has been suggested to be a risk factor for pancreatic cancer, it can also develop as a result of pancreatic cancer.

Pancreatic cancer is thought to have a different nature in patients with DM as a background factor (long-standing DM) and in those with DM as a consequence of cancer (short-term DM). Previous studies from the United States

\footnotetext{
${ }^{1}$ Department of Hepatobiliary and Pancreatic Oncology, National Cancer Center Hospital, Japan, ${ }^{2}$ Keio University Graduate School of Medicine, Japan, ${ }^{3}$ Biostatistics Division, Center for Research Administration and Support, National Cancer Center, Japan, ${ }^{4}$ Department of Hepatobiliary and Pancreatic Oncology, National Cancer Center Hospital East, Japan and ${ }^{5}$ Department of General Internal Medicine, National Cancer Center Hospital, Japan

Received: December 17, 2018; Accepted: March 18, 2019; Advance Publication by J-STAGE: June 7, 2019

Correspondence to Dr. Ken Ohashi, keohashi@ncc.go.jp
} 
and United Kingdom showed that pancreatic cancer patients with long-standing DM had worse survival than nondiabetic patients, while patients with short-term DM had a similar survival to nondiabetic patients $(1,2)$. However, these studies included a heterogeneous patient population with different stages of cancer and did not consider differences in treatment. Thus, the impact of the duration of DM on survival has not been sufficiently studied, especially in patients receiving chemotherapy for metastatic disease, which is the most common extent of disease at presentation (3).

We conducted a retrospective study of a homogeneous patient population with metastatic pancreatic cancer treated with gemcitabine monotherapy to evaluate the impact of the duration of DM on the outcomes of chemotherapy using data from two different hospital sites.

\section{Materials and Methods}

\section{Patients}

We reviewed the medical records of patients with metastatic pancreatic cancer. The selection criteria were as follows: 1) pathologically proven metastatic adenocarcinoma of the pancreas; 2) initiation of gemcitabine monotherapy as first-line treatment at the National Cancer Center Hospital (Tokyo, Japan) ("Tokyo") or at the National Cancer Center Hospital East (Kashiwa, Japan) ("Kashiwa") before the introduction of combination therapies [oxaliplatin/irinotecan/ fluorouracil/leucovorin, or gemcitabine/nanoparticle albuminbound paclitaxel (nab-PTX), or gemcitabine/erlotinib] as standard treatments; and 3) no previous curative surgery for pancreatic cancer. We selected single gemcitabine monotherapy regimen to enable a uniform assessment of the patient outcomes. The institutional review board approved the study (No. 2016-233). Written informed consent was not obtained because of the retrospective nature of this study. The study design of this study was publicized via the web page of the hospitals, according to the Ethics Guidelines for Medical and Health Research in Japan.

\section{Treatment}

Patients received intravenous infusions of gemcitabine $\left(1,000 \mathrm{mg} / \mathrm{m}^{2}\right.$ of body surface area) over 30 minutes on days 1,8 , and 15 of each 28-day cycle. The dosing was adjusted according to the adverse events (AEs) and the physician's judgment. Treatment was continued until the development of progressive disease or unacceptable toxicity, or until the patient expressed a wish to discontinue treatment.

\section{Assessment}

DM was defined as either a diagnosis of DM documented in the patient's medical records or at least one serum glucose measurement of $\geq 200 \mathrm{mg} / \mathrm{dL}$. We used the serum glucose levels because data on finger-stick levels were not available. As HbA1c was not routinely measured at "Kashiwa," it was not included in the definition of diabetes. The duration of DM was determined based on medical records. Patients with DM of uncertain duration were excluded from the analysis. As in a previous study (1), short DM was defined as DM for a duration of $<4$ years, while long DM was defined as DM with a duration of $\geq 4$ years. This previous study used four years as a cutoff based on reports of an association between an increased incidence of pancreatic cancer and new-onset DM, classified as DM for less than three to five years (4-6). We assessed the tumor response according to the Response Evaluation Criteria in Solid Tumors (RECIST), version 1.1, and based on computed tomography (CT) scans. Generally, the baseline scan covered the neck though the pelvis and subsequent scans included the areas of the lesions detected on the baseline scan. CT scans were obtained every other cycle, and as needed. Confirmation of a response was not required. Stable disease (SD) as the best response required a duration of at least four weeks. The response rate (RR) and disease control rate (DCR) were defined as the proportions of patients who achieved at least a partial response (PR) and SD, respectively. Progression-free survival (PFS) was defined as the time from the initiation of gemcitabine monotherapy until clinical or objective disease progression, or death. Overall survival (OS) was defined as the time from the initiation of gemcitabine monotherapy until death. AEs were assessed using the Common Terminology Criteria for Adverse Events (CTCAE), version 4.0.

\section{Statistical analysis}

The present study was designed to compare the clinical outcomes (survival outcomes, tumor response, and AEs) between groups with different durations of DM (no DM, short DM, and long DM). Data were analyzed for the combined overall population (both hospital sites) and separately for each hospital population. These hospital-specific analyses were conducted to confirm the reproducibility of the outcomes in different environments. Comparisons of efficacy and safety were conducted using the no DM group as a reference. Categorical variables were compared using Fisher's exact test. The Kruskal-Wallis test was used for comparisons of continuous variables among the three groups. Survival curves were estimated using the Kaplan-Meier method; differences between groups were assessed using the log-rank test. Hazard ratios (HRs) and $95 \%$ confidence intervals (CIs) were estimated using a Cox proportional hazards model with stratification according to site. Sensitivity analyses using different cut-off values for the duration of DM (three and five years) and multivariate analyses adjusted according to the baseline characteristics selected by the univariate analyses were also conducted. Based on a previous study (1), the HRs for PFS and OS in the long DM group using antidiabetic medication in comparison to the no DM group were estimated and adjusted for selected baseline characteristics. Since a linear relationship between body mass index (BMI) and survival outcome was not assumed, the comparison was made using categories with a BMI cutoff value of 25.0 $\mathrm{kg} / \mathrm{m}^{2}$, as previously reported (1). The criterion for the selec- 


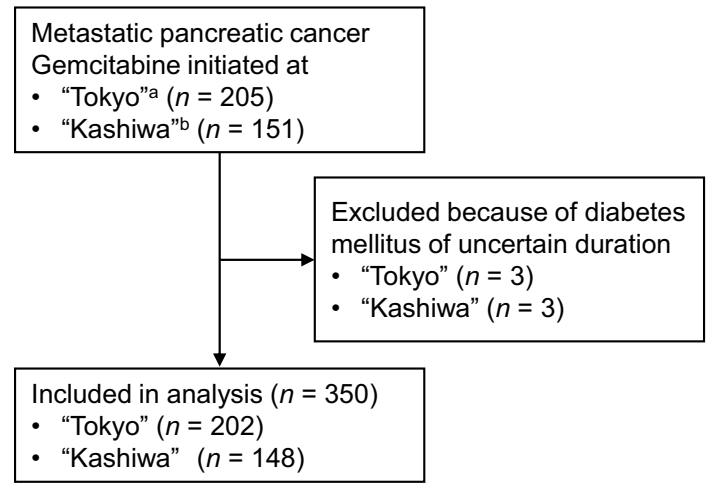

Figure 1. Flow diagram of patient selection. ${ }^{\text {aBetween Janu- }}$ ary 2008-December 2013. 'between January 2008-December 2011.

tion of baseline characteristics for multivariate analyses was a $p$ value of $<0.10$. Otherwise, $p$ values of $<0.05$ were considered to indicate statistical significance. All $\mathrm{p}$ values were two-sided. Patients with missing data for any of the variables or with a carbohydrate antigen 19-9 (CA19-9) level of $<1 \mathrm{U} / \mathrm{mL}$ were excluded from analyses requiring these variables. The analyses were performed using EZR (Saitama Medical Center, Jichi Medical University, Saitama, Japan), which is a graphical user interface for $\mathrm{R}$ (The R Foundation for Statistical Computing, Vienna, Austria) (7).

\section{Results}

\section{Patients}

A total of 356 patients (205 patients from "Tokyo" and 151 patients from "Kashiwa") started gemcitabine monotherapy between 2008 and 2013 at "Tokyo" (before the introduction of oxaliplatin/irinotecan/fluorouracil/leucovorin therapy) and between 2008 and 2011 at "Kashiwa" (before the introduction of gemcitabine/erlotinib therapy). Among these, three patients from each site had DM of uncertain duration and were excluded from the analyses, leaving 350 patients (202 from "Tokyo" and 148 from "Kashiwa") in the study (Fig. 1). Among the patients included in the study, $24.9 \%$ and $12.9 \%$ were classified into the Short DM and long DM groups, respectively ("Tokyo", $22.8 \%$ and 12.4\%; "Kashiwa", $27.7 \%$ and $13.5 \%$ ). Besides assumed secondary DM (due to pancreatic cancer) among patients in the short DM group, the etiology of DM was type $2 \mathrm{DM}$ in $93.3 \%$ of patients in the long DM group (the etiology was unclear in the other patients in the long DM group). No statistically significant differences were observed in the baseline characteristics of the three groups, with the exception of BMI, which was highest in the long DM group (Table 1). Of note, metformin, and insulin (oral hypoglycemic agents) were administered to 29 (65.9\%), $12(27.3 \%)$, and 18 (40.9\%) patients, respectively, in the long DM group. The median follow-up period was 5.6 months (range, 0.1-62.8 months).

\section{Survival outcomes}

The PFS and OS of the long DM and short DM groups of the combined overall population (both hospitals) were compared with those in the no DM group (Fig. 2). In the long DM group, the HR for PFS was 1.44 (95\% CI, 1.02$2.02 ; \mathrm{p}=0.027)$ and the HR for OS was 1.33 (95\% CI, 0.93$1.89 ; \mathrm{p}=0.081)$ in comparison to the no DM group. In the short DM group, the HR for PFS was 1.12 (95\% CI, 0.851.46 ; $\mathrm{p}=0.426)$ and the HR for OS was 1.06 (95\% CI, 0.81$1.40 ; \mathrm{p}=0.678)$ in comparison to the no DM group. In a multivariate analysis, the HRs for PFS and OS were adjusted according to baseline characteristics selected using a univariate analysis [ECOG PS, the number of organs involved, and the types of organs involved (lymph node and liver) for PFS; ECOG PS, the number of organs involved, the types of organs involved (lymph node and liver), and the CA19-9 level for OS; Supplementary material 1]. The adjustment did not have a large impact on the HRs. The adjusted HRs for PFS and OS were 1.33 (95\% CI, 0.94-1.89; $\mathrm{p}=0.103$ ) and 1.37 (95\% CI, 0.95-1.98; $\mathrm{p}=0.095)$, respectively, in the long DM group, and 1.12 (95\% CI, 0.85-1.47; $\mathrm{p}=0.410)$ and 1.10 (95\% CI, 0.82-1.46; p=0.533), respectively, in the short DM group. There were no marked changes in the HRs when cut-off values of three or five years were used to define the duration of DM in the univariate and multivariate analyses (Table 2).

Supplementary material 2, 3 show the Kaplan-Meier curves for PFS and OS for each hospital site. In the "Tokyo" population, the PFS in the long DM group was significantly worse than that in the no DM group (HR, 1.54; 95\% CI, 0.98-2.44; $\mathrm{p}=0.048$ compared with the no DM group). The trend toward a worse outcome in the long DM group was also seen for OS (HR, 1.32; 95\% CI, 0.82-2.14; p= 0.242 in comparison to the no DM group). In the short DM group, the PFS and OS values were similar to those observed in the no DM group (PFS: HR, 1.09; 95\% CI, 0.76$1.56 ; \mathrm{p}=0.662$ in comparison to the no DM group; OS: HR, $0.95 ; 95 \% \mathrm{CI}, 0.65-1.39 ; \mathrm{p}=0.791$ in comparison to the no DM group). In "Kashiwa," there was also a trend toward a shorter PFS and OS in the long DM group in comparison to the no DM group (PFS: HR, 1.33; 95\% CI, 0.80-2.21; $\mathrm{p}=$ 0.256 ; OS: HR, $1.34,95 \%$ CI, $0.80-2.26$; $\mathrm{p}=0.190$ in comparison to the no DM group). The PFS and OS in the short DM group in the "Kashiwa" population fell between those in the no DM and long DM groups (PFS: HR, 1.15; 95\% CI, 0.77-1.72; $\mathrm{p}=0.479$; OS: HR, 1.21, 95\% CI, 0.81-1.80; $\mathrm{p}=0.364$ in comparison to the no DM group). A trend toward a worse PFS and OS in the long DM group was also seen in multivariate analyses conducted separately for each site (Supplementary material 4).

The HRs for PFS and OS in the long DM group according to the use of antidiabetic medication are shown in Supplementary material 5. A univariate analysis revealed that users of oral medication in the long DM group had significantly reduced PFS in comparison to the no DM group (HR, 
Table 1. Patient Characteristics.

\begin{tabular}{|c|c|c|c|c|c|}
\hline Characteristics & & No DM & Short DM & Long DM & $\mathrm{p}$ value \\
\hline $\mathrm{n}$ & & 218 & 87 & 45 & \\
\hline Age & Median [range] & $66[32-86]$ & $66[44-84]$ & $66[50-83]$ & 0.647 \\
\hline \multirow[t]{2}{*}{ Sex } & Male (\%) & $116(53.2)$ & $51(58.6)$ & $32(71.1)$ & 0.077 \\
\hline & Female (\%) & $102(46.8)$ & $36(41.4)$ & $13(28.9)$ & \\
\hline \multirow[t]{4}{*}{ ECOG PS } & 0 & $113(51.8)$ & $48(55.2)$ & $24(53.3)$ & 0.564 \\
\hline & 1 & $91(41.7)$ & $37(42.5)$ & $18(40.0)$ & \\
\hline & 2 & $12(5.5)$ & $1(1.1)$ & $2(4.4)$ & \\
\hline & NA & $2(0.9)$ & $1(1.1)$ & $1(2.2)$ & \\
\hline \multirow[t]{3}{*}{ Location } & Head $(\%)$ & 75 (34.4) & $32(36.8)$ & $13(28.9)$ & 0.270 \\
\hline & Body $(\%)$ & $81(37.2)$ & $35(40.2)$ & $13(28.9)$ & \\
\hline & Tail (\%) & $62(28.4)$ & $20(23.0)$ & $19(42.2)$ & \\
\hline \multirow[t]{3}{*}{ No. of organs involved } & $<2(\%)$ & $123(56.4)$ & $52(59.8)$ & $27(60.0)$ & 0.843 \\
\hline & $\geq 2(\%)$ & $95(43.6)$ & $35(40.2)$ & $18(40.0)$ & \\
\hline & Median [range] & $1[1-5]$ & $1[1-5]$ & $1[1-4]$ & 0.824 \\
\hline \multirow[t]{5}{*}{ Organs involved } & Lymph node (\%) & $75(34.4)$ & $29(33.3)$ & $16(35.6)$ & 0.958 \\
\hline & Liver (\%) & 159 (72.9) & 64 (73.6) & $38(84.4)$ & 0.281 \\
\hline & Lung (\%) & $39(17.9)$ & $17(19.5)$ & $11(24.4)$ & 0.574 \\
\hline & Peritoneum (\%) & $78(35.8)$ & $27(31.0)$ & $10(22.2)$ & 0.193 \\
\hline & Bone $(\%)$ & $5(2.3)$ & $2(2.3)$ & $0(0.0)$ & 0.744 \\
\hline \multirow[t]{5}{*}{ CA $19-9^{b}$} & Normal (\%) & $20(9.2)$ & $13(14.9)$ & $5(11.1)$ & 0.482 \\
\hline & Elevated, $<59 \times$ ULN (\%) & $82(37.6)$ & $26(29.9)$ & $16(35.6)$ & \\
\hline & Elevated, $\geq 59 \times$ ULN (\%) & $102(46.8)$ & $47(54)$ & $23(51.1)$ & \\
\hline & NA & $14(6.4)$ & $1(1.1)$ & $1(2.2)$ & \\
\hline & Median [range] & $2,205[2-1,861,000]$ & $3,081[1-2,379,000]$ & $2,418[3-312,700]$ & 0.933 \\
\hline \multirow[t]{2}{*}{ Biliary stent/tube } & Yes $(\%)$ & $43(19.7)$ & 19 (21.8) & $9(20.0)$ & 0.925 \\
\hline & No $(\%)$ & $175(80.3)$ & $68(78.2)$ & $36(80.0)$ & \\
\hline \multirow[t]{4}{*}{$\operatorname{BMI}\left(\mathrm{kg} / \mathrm{m}^{2}\right)$} & $<25.0$ & 195 (89.4) & $76(87.4)$ & $33(73.3)$ & 0.008 \\
\hline & $\geq 25.0$ & $21(9.6)$ & $11(12.6)$ & $12(26.7)$ & \\
\hline & NA & $2(0.9)$ & $0(0.0)$ & $0(0.0)$ & \\
\hline & Median [range] & $20.7[14.2-30.3]$ & $21.4[14.9-30.3]$ & $22.5[16.0-33.6]$ & 0.015 \\
\hline \multirow[t]{3}{*}{ DM medication } & Oral (\%) & - & $39(45.3)$ & $29(65.9)$ & NA \\
\hline & Metformin (\%) & - & $2(2.3)$ & $12(27.3)$ & NA \\
\hline & Insulin (\%) & - & 17 (19.8) & $18(40.9)$ & NA \\
\hline
\end{tabular}

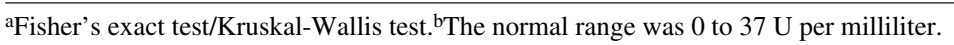

DM: diabetes mellitus, ECOG PS: Eastern Cooperative Oncology Group Performance Status, NA: not assessed, CA19-9: carbohydrate antigen 19-

9, ULN: upper limit of normal, BMI: body mass index

$1.60 ; 95 \%$ CI, 1.05-2.44; $\mathrm{p}=0.028$ ) and a multivariate analysis revealed a trend toward worse PFS (HR, 1.46; 95\% CI, 0.95-2.22; $\mathrm{p}=0.083)$.

PFS and OS were assessed according to HbA1c among patients in "Tokyo," where the HbA1c levels of patients with metastatic pancreatic cancer were routinely measured at the start of chemotherapy. The unadjusted and adjusted HRs per $1 \%$ increase in $\mathrm{HbA1c}$ for PFS were 1.05 (CI, 0.95$1.16 ; \mathrm{p}=0.341)$ and $1.00(\mathrm{CI}, 0.9-1.11 ; \mathrm{p}=0.963)$, respectively; those for OS were 0.98 (CI, 0.88-1.09; $\mathrm{p}=0.706$ ) and 0.94 (CI, 0.84-1.05; $\mathrm{p}=0.291$ ), respectively.

\section{Tumor response}

The RRs in the no DM, short DM, and long DM groups were $5.0 \%, 2.3 \%$, and $11.1 \%$, respectively (no DM vs. short DM: $p=0.362$; no DM vs. long DM: $p=0.164)$; the DCRs were 51.4\%, 50.6\%, and 37.8\%, respectively (no DM vs. short DM: $\mathrm{p}=0.900$; no DM vs. long DM: $\mathrm{p}=0.104$ ).

\section{Safety}

The AEs in the overall population are shown in Supplementary material 6. The incidence of AEs did not differ to a statistically significant extent when the AEs of the long DM and short DM groups were compared with those of the no DM group. AEs leading to treatment discontinuation were seen in the no DM [ 4 patients (grade 3 pneumonitis, grade 2 pneumonitis, grade 2 rash, and grade 1 nausea; $1.8 \%$ )] and short DM groups [1 patient (grade 2 pneumonitis; $1.1 \%$ )]. No patients in the long DM group $(0.0 \%)$ developed AEs leading to treatment discontinuation. 

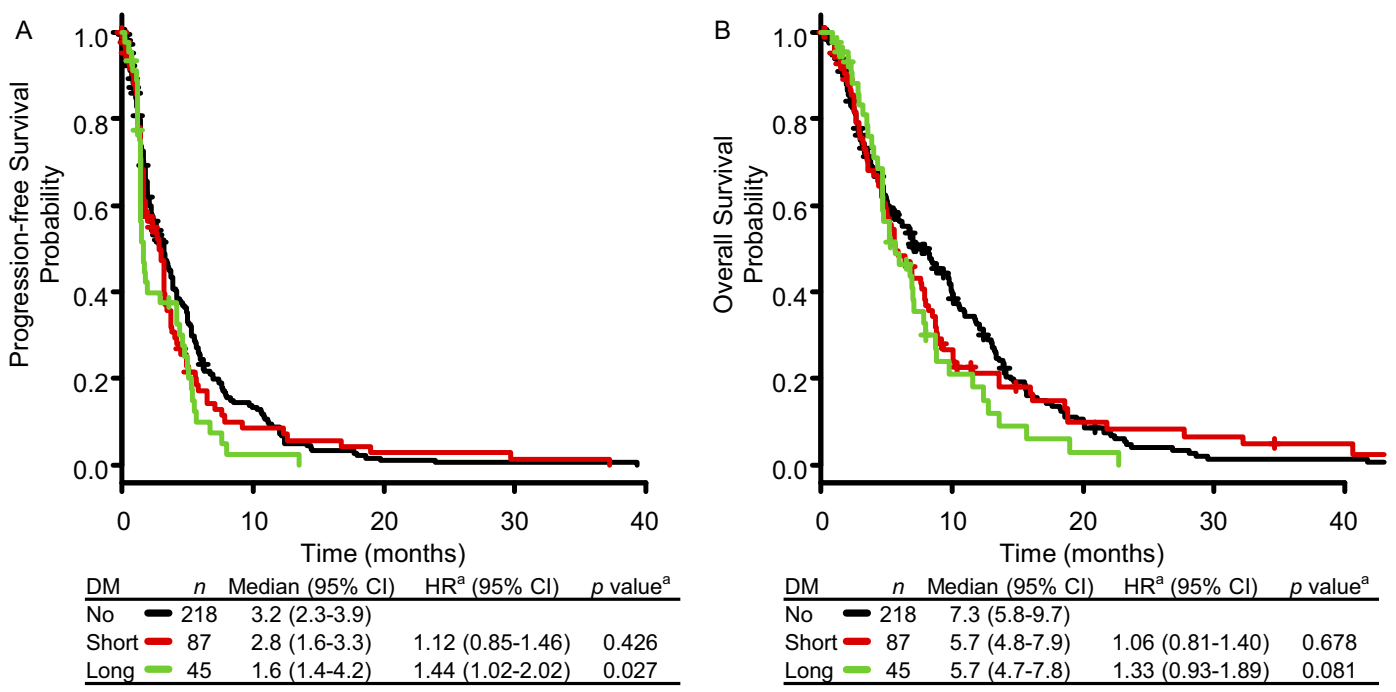

Figure 2. Kaplan-Meier curves for (a) progression-free survival and (b) overall survival of the overall population. ${ }^{\text {IIn }}$ comparison to no DM. DM: diabetes mellitus, HR: hazard ratio, CI: confidence interval

Table 2. Sensitivity Analysis with Different Cutoffs for Diabetes Mellitus Duration and Multivariate Analysis for Progression-free Survival and Overall Survival in the Overall Population.

\begin{tabular}{cccccc}
\hline & \multicolumn{3}{c}{ Univariate } & Multivariate \\
\hline DM & Cutoff & HR $(95 \% \text { CI })^{\mathrm{a}}$ & p value & HR $(95 \% \text { CI })^{\mathrm{a}}$ & p value \\
\hline \multirow{5}{*}{ Short DM } & 3 years & $1.13(0.86-1.49)$ & 0.378 & $1.13(0.85-1.49)$ & 0.395 \\
& 4 years & $1.12(0.85-1.46)$ & 0.419 & $1.12(0.85-1.47)$ & 0.410 \\
& 5 years & $1.10(0.85-1.43)$ & 0.463 & $1.12(0.86-1.47)$ & 0.388 \\
\hline Long DM & 3 years & $1.36(0.98-1.89)$ & 0.062 & $1.30(0.93-1.81)$ & 0.129 \\
& 4 years & $1.44(1.02-2.02)$ & 0.035 & $1.33(0.94-1.89)$ & 0.103 \\
& 5 years & $1.55(1.09-2.22)$ & 0.016 & $1.36(0.94-1.96)$ & 0.100 \\
\hline \multirow{2}{*}{ Short DM } & 3 years & $1.10(0.83-1.46)$ & 0.512 & $1.13(0.84-1.52)$ & 0.403 \\
& 4 years & $1.06(0.81-1.40)$ & 0.674 & $1.10(0.82-1.46)$ & 0.533 \\
& 5 years & $1.08(0.82-1.41)$ & 0.590 & $1.13(0.85-1.49)$ & 0.397 \\
\hline Long DM & 3 years & $1.21(0.87-1.69)$ & 0.263 & $1.25(0.88-1.77)$ & 0.215 \\
& 4 years & $1.33(0.93-1.89)$ & 0.115 & $1.37(0.95-1.98)$ & 0.095 \\
& 5 years & $1.32(0.91-1.92)$ & 0.144 & $1.31(0.88-1.93)$ & 0.183 \\
\hline
\end{tabular}

${ }^{\mathrm{a} C o x}$ proportional hazards model. No DM as reference. In multivariate analyses, hazard ratios were adjusted for Eastern Cooperative Oncology Group Performance Status, number of organs involved, and types of organs involved (lymph node and liver) for progression-free survival, and Eastern Cooperative Oncology Group Performance Status, number of organs involved, types of organs involved (lymph node and liver), and CA 19-9 level for overall survival.

DM: diabetes mellitus, HR: hazard ratio, CI: confidence interval, PFS: progression-free survival, OS: overall survival

\section{Discussion}

In this retrospective study, we evaluated the outcomes of gemcitabine monotherapy according to the duration of DM in metastatic pancreatic cancer patients treated at two different hospital sites. A trend toward a shorter PFS and OS was seen in the long DM group in comparison to the no DM group. This trend remained unchanged in a sensitivity analysis with different cut-off values for duration of DM and in a multivariate analysis adjusted for the patients' baseline characteristics, as well as in analyses conducted separately for each site. Within the long DM group, the use of oral hypoglycemic agents seemed to be associated with a worse PFS. 
There was no apparent trend in the difference in tumor response or AEs among the three groups.

In this study, the long DM group had worse PFS and OS than the no DM group. Although it was not reflected in the tumor response, this result is in line with previous studies reporting similar results in pancreatic cancer patients in Western populations $(1,2)$. In East Asian patients, who may have a different diabetic nature from Western patients [such as an increased risk of DM at a lower BMI and a stronger association with cancer (8-10)], one study reported that long-standing DM and short-term DM were associated with worse survival outcomes in comparison to nondiabetic patients (11). However, the interpretation of this study's results is not straightforward because of the non-uniform patient population, in terms of tumor histology, stage, and treatment. Furthermore, the study may have included a bias due to the assessment of DM, which continued throughout the course of the disease. Thus, the current study is of additional importance in that it provides data obtained from East Asian patients, who have not been sufficiently studied. The evaluation according to antidiabetic medication was limited by the small number of users of such medications (Supplementary material 5) and further assessment is needed. Nevertheless, the survival outcomes of patients with longstanding DM who received oral hypoglycemic agents seemed to be worse than those of patients without DM, as previously reported (1); however, metformin, which has been suggested to show a protective effect against cancer (12), did not have a significant influence the survival outcomes. Oral hypoglycemics showed higher univariate/ multivariate hazard ratios for PFS than insulin as point estimates (Table S3: oral hypoglycemics, 1.60/1.46; insulin, $1.32 / 1.14)$. As hyperglycemia may be associated with chemoresistance and cancer progression $(13,14)$, one possibility is that obtaining tight glycemic control using insulin reduces the risk of cancer progression; however, this remains a hypothesis and the largely overlapping confidence interval requires cautious interpretation. Regarding the magnitude of the impact of long-standing DM, in comparison to the no DM group, the HRs for PFS and OS in the long DM group were similar to those of patients who received gemcitabine as a monotherapy rather than as a combination therapy with nab-PTX (calculated as the reciprocals of the HRs of combination therapy in comparison to monotherapy: 1/0.69 $=1.45$ and $1 / 0.72=1.39$ for PFS and OS, respectively) (15). Thus, long-standing DM seems to have a negative effect equivalent to the difference between monotherapy with gemcitabine and combination therapy with nab-PTX.

In the short DM group, the PFS and OS seemed to differ between the two hospital sites. Previous reports on the survival of pancreatic cancer patients without DM and those with DM of different durations have reported both similar survival outcomes and different survival outcomes according to the DM status $(1,2,11,16,17)$. The differences between studies may be due to differences in the methods used to detect DM. In the current study, the proportion of patients be- longing to the short DM group appeared to be larger in the "Kashiwa" population, implying that a between-site difference in the detection of DM, such as the timing of glucose measurement, might have existed. As long-standing DM seems to have a negative impact on survival and differences in the detection of DM may cause different outcomes, the duration of DM should be assessed in a uniform manner and should be carefully documented at baseline in clinical studies.

The duration of DM did not appear to affect the safety of gemcitabine monotherapy. In this study, the frequencies of AEs or AEs leading to treatment discontinuation did not differ significantly among the different groups defined according to the duration of DM. This finding suggests that the poor survival outcomes in patients with long-standing DM were not due to insufficient treatment caused by AEs. Rather, they were more likely to be due to a lower efficacy of treatment in such patients.

Because the relationship between DM and cancer mortality is complex, the etiological association requires cautious evaluation. One article addressed nine issues to consider when assessing the relationship between DM and cancer mortality: 1) cancer screening use; 2) stage at the diagnosis; 3) cancer treatment selection; 4) cancer treatment complications and failures; 5) peri-treatment (perioperative) mortality; 6) competing risks for long-term mortality; 7) effects of type 2 DM on anti-cancer therapies; 8) effects of glucoselowering treatments on the cancer outcome and 9) differences in tumor biology (18). As the current study assessed nonsurgical patients with the same stage, who received the same treatment, and in whom long-term survival was less likely to be achieved in comparison to patients with other cancer types, the relevant points that should be considered are 4), 7), 8), and 9). In relation to 9), there have been studies reporting the association between the development of pancreatic cancer and DM-related hormones, such as adiponectin and leptin $(19,20)$, as well as one study suggesting an increased risk of pancreatic cancer in patients with a single-nucleotide polymorphism related to DM (21). However, there continues to be a paucity of data, and the mechanism responsible for the poor survival outcomes in patients with long-standing DM remains to be elucidated. Otherwise, all of the abovementioned relevant points were covered in this study, which makes the presence of biases associated with the link between DM and pancreatic cancer less likely.

Our study was associated with some limitations. First, it was a retrospective study with a relatively small sample size. Only grade 3 or worse AEs were assessed because of insufficient documentation regarding less severe AEs. Although there was a trend toward a worse PFS and OS in the long DM group, statistically significant differences were only observed for PFS in the overall and "Tokyo" populations, probably because of the small sample sizes. Second, the glucose levels were measured using serum samples obtained with no regard to the timing of meals. Thus, the no DM group might have included patients with undetected DM. 
Third, we only studied patients who received gemcitabine monotherapy. Patients receiving other chemotherapy regimens should also be studied. Furthermore, the follow-up time may have been inadequate for observing survival outcomes. The limited follow-up time is likely due to the large proportion of patients who moved to another hospital from our institutions in order to focus on the best supportive care and who were consequently lost to follow-up, reflecting the nature of the clinical practice of the study sites. Nonetheless, the current study evaluated several factors that had not been necessarily assessed in previous studies. Clinically important baseline characteristics of patients with pancreatic cancer, such as PS and the involved organs, were included in the analyses. This study assessed not only overall survival as an outcome, but also other efficacy outcomes, such as the tumor response and PFS. Moreover, we collected data from a homogeneous population of patients with regard to the extent of disease (metastatic disease) and the chemotherapy line and regimen. Furthermore, data from two different hospital sites were collected, and the results were confirmed.

In conclusion, this study suggests that long-standing DM had an adverse effect on the PFS and OS of patients with metastatic pancreatic cancer. The incorporation of longstanding DM in evaluations of baseline characteristics in clinical studies should therefore be considered. The mechanism responsible for the adverse effect of long-standing DM on the survival outcomes remains to be elucidated.

\section{Author's disclosure of potential Conflicts of Interest (COI).}

Sakura Iizumi: Employment, AstraZeneca. Masafumi Ikeda: Honoraria, Taiho; Research funding, Yakult, Taiho, AstraZeneca, Nano Carrier, Eli Lilly, Baxter and Merck Serono. Yasunari Sakamoto: Research funding, Sumitomo Dainippon Pharma and Pfizer. Shunsuke Kondo: Research funding, Pfizer, Merck Serono, AstraZeneca, Bayer and Eli Lilly. Chigusa Morizane: Employment, AstraZeneca, Yakult, Novartis and Taiho; Honoraria, Pfizer, Novartis, Yakult, Eli Lilly, Nobelpharma and FUJIFILM RI Pharma. Shuichi Mitsunaga: Honoraria, Ono and Toray; Research funding, Bayer, Chugai, Merck Serono, ASLAN and Ajinomoto.

\section{Acknowledgement}

We would like to express our sincere thanks to all patients and investigators.

\section{References}

1. Yuan C, Rubinson DA, Qian ZR, et al. Survival among patients with pancreatic cancer and long-standing or recent-onset diabetes mellitus. J Clin Oncol 33: 29-35, 2015.

2. Hwang A, Narayan V, Yang YX. Type 2 diabetes mellitus and survival in pancreatic adenocarcinoma: a retrospective cohort study. Cancer 119: 404-410, 2013.

3. National Cancer Institute. Contents of the SEER Cancer Statistics Review (CSR), 1975-2014 [Internet]. [cited 2018 Dec 15]. Avail- able from: https://seer.cancer.gov/csr/1975_2014/sections.html

4. Chari ST, Leibson CL, Rabe KG, et al. Pancreatic cancerassociated diabetes mellitus: prevalence and temporal association with diagnosis of cancer. Gastroenterology 134: 95-101, 2008.

5. Ben Q, Xu M, Ning X, et al. Diabetes mellitus and risk of pancreatic cancer: a meta-analysis of cohort studies. Eur J Cancer 47: 1928-1937, 2011.

6. Huxley R, Ansary-Moghaddam A, Berrington de Gonzalez A, Barzi F, Woodward M. Type-II diabetes and pancreatic cancer: a meta-analysis of 36 studies. Br J Cancer 92: 2076-2083, 2005.

7. Kanda Y. Investigation of the freely available easy-to-use software 'EZR' for medical statistics. Bone Marrow Transplant 48: 452458, 2013.

8. Yoon KH, Lee JH, Kim JW, et al. Epidemic obesity and type 2 diabetes in Asia. Lancet 368: 1681-1688, 2006.

9. Noto H, Tsujimoto T, Noda M. Significantly increased risk of cancer in diabetes mellitus patients: a meta-analysis of epidemiological evidence in Asians and non-Asians. J Diabetes Investig 3: 2433, 2012.

10. Hotta N, Nakamura J, Iwamoto Y, et al. Causes of death in Japanese diabetics: a questionnaire survey of 18,385 diabetics over a 10-year period. J Diabetes Investig 1: 66-76, 2010.

11. Wakasugi H, Funakoshi A, Iguchi H. Clinical observations of pancreatic diabetes caused by pancreatic carcinoma, and survival period. Int J Clin Oncol 6: 50-54, 2001.

12. Decensi A, Puntoni M, Goodwin P, et al. Metformin and cancer risk in diabetic patients: a systematic review and meta-analysis. Cancer Prev Res (Phila) 3: 1451-1461, 2010.

13. Zeng L, Biernacka KM, Holly JM, et al. Hyperglycaemia confers resistance to chemotherapy on breast cancer cells: the role of fatty acid synthase. Endocr Relat Cancer 17: 539-551, 2010.

14. Saengboonmee C, Seubwai W, Pairojkul C, Wongkham S. High glucose enhances progression of cholangiocarcinoma cells via STAT3 activation. Sci Rep 6: 18995, 2016.

15. Von Hoff DD, Ervin T, Arena FP, et al. Increased survival in pancreatic cancer with nab-paclitaxel plus gemcitabine. N Engl J Med 369: 1691-1703, 2013.

16. Chu CK, Mazo AE, Goodman M, et al. Preoperative diabetes mellitus and long-term survival after resection of pancreatic adenocarcinoma. Ann Surg Oncol 17: 502-513, 2010.

17. Dandona M, Linehan D, Hawkins W, Strasberg S, Gao F, Wang-Gillam A. Influence of obesity and other risk factors on survival outcomes in patients undergoing pancreaticoduodenectomy for pancreatic cancer. Pancreas 40: 931-937, 2011.

18. Renehan AG, Yeh HC, Johnson JA, Wild SH, Gale EA, Moller H. Diabetes and cancer (2): evaluating the impact of diabetes on mortality in patients with cancer. Diabetologia 55: 1619-1632, 2012.

19. Babic A, Bao Y, Qian ZR, et al. Pancreatic cancer risk associated with prediagnostic plasma levels of leptin and leptin receptor genetic polymorphisms. Cancer Res 76: 7160-7167, 2016.

20. Bao Y, Giovannucci EL, Kraft P, et al. A prospective study of plasma adiponectin and pancreatic cancer risk in five US cohorts. J Natl Cancer Inst 105: 95-103, 2013.

21. Prizment AE, Gross M, Rasmussen-Torvik L, Peacock JM, Anderson KE. Genes related to diabetes may be associated with pancreatic cancer in a population-based case-control study in Minnesota. Pancreas 41: 50-53, 2012.

The Internal Medicine is an Open Access journal distributed under the Creative Commons Attribution-NonCommercial-NoDerivatives 4.0 International License. To view the details of this license, please visit (https://creativecommons.org/licenses/ by-nc-nd/4.0/).

(C) 2019 The Japanese Society of Internal Medicine Intern Med 58: 2435-2441, 2019 